\title{
The use of aerosol data in Auger Fluorescence Detector analysis
}

\author{
Bruce R. Dawson ${ }^{1, *}$ for the Pierre Auger Collaboration ${ }^{2, * *, * * *}$ \\ ${ }^{1}$ Department of Physics, University of Adelaide, Adelaide, S.A. 5005, Australia \\ ${ }^{2}$ Observatorio Pierre Auger, Av. San Martín Norte 304, 5613 Malargüe, Argentina
}

\begin{abstract}
.
The Pierre Auger Observatory's Fluorescence Detector (FD) consists of 27 telescopes arranged in four sites around the perimeter of the 3000 square kilometre Surface Detector (SD). Cosmic ray extensive air showers are viewed via the nitrogen fluorescence light they induce in the atmosphere. Careful treatment of light attenuation processes must be made, especially given that some showers are viewed at distances in excess of $30 \mathrm{~km}$. Of particular importance is the attenuation due to scattering by aerosol particles, a challenging topic given that aerosol concentrations can vary on time-scales of hours. At the Auger Observatory, the vertical distribution of aerosols is measured hourly with a series of bi-static lidar systems (consisting of central laser facilities and each of the FD sites), and three times per night with a Raman lidar system. In this contribution we describe the use of aerosol profiles in the analysis of air shower data, in particular in the estimation of the cosmic ray primary energy, and the depth of shower maximum, $X_{\max }$. We also demonstrate how statistical and systematic uncertainties in the aerosol concentrations propagate through to a contribution to energy and $X_{\max }$ uncertainties.
\end{abstract}

\section{Introduction}

An observatory for ultra-high energy cosmic rays (UHECRs) requires an enormous collecting area and reliable methods for assigning arrival directions, energy and estimates of primary mass. The Pierre Auger Observatory satisfies these criteria with a surface detector (SD) covering an area of 3000 square kilometres, and a complementary fluorescence detector (FD) which views the atmosphere above the surface array during clear, moonless nights. The Observatory is located in western Argentina in the province of Mendoza on an elevated plain with an altitude of approximately $1400 \mathrm{~m} \mathrm{[1].}$

The atmosphere is both the target for the incoming primary cosmic rays and the detection medium. The extensive air shower (EAS) initiated by the cosmic ray has a footprint at ground level of tens of square kilometres allowing for SD estimates of arrival direction and energy. The longitudinal development of the EAS may be observed with the FD via nitrogen fluorescence light. This rather weak light signal (at least compared with Cherenkov light) is emitted isotropically and in proportion to the ionisation energy deposited by the shower in the atmosphere. Of importance to Auger is the emission band from 300$400 \mathrm{~nm}$ accessible to the photomultipliers of the FD telescopes [2]. The most energetic EAS can be observed in fluorescence light at distances even beyond $30 \mathrm{~km}$.

There is no significant atmospheric absorption of this wavelength band in the troposphere, the layer containing the bulk of the EAS. (Ozone absorbs in this band, but its

\footnotetext{
*e-mail: bruce.dawson@adelaide.edu.au

**e-mail: auger_spokespersons@fnal.gov

***Author list: http://www.auger.org/archive/authors_2018_09.html
}

effect is only important at higher altitudes). This leaves scattering as the prime mechanism for attenuation of the fluorescence light - scattering by molecules (Rayleigh scattering) and by aerosols. Attenuation by Rayleigh scattering is the strongest, but this can be calculated precisely enough with a model of the atmospheric density profile (available, for example, every three-hours via the Global Data Assimilation System, GDAS [3]). Aerosol scattering is weaker but nonetheless important for precise EAS measurements (see Figure 1), with the added difficulty that

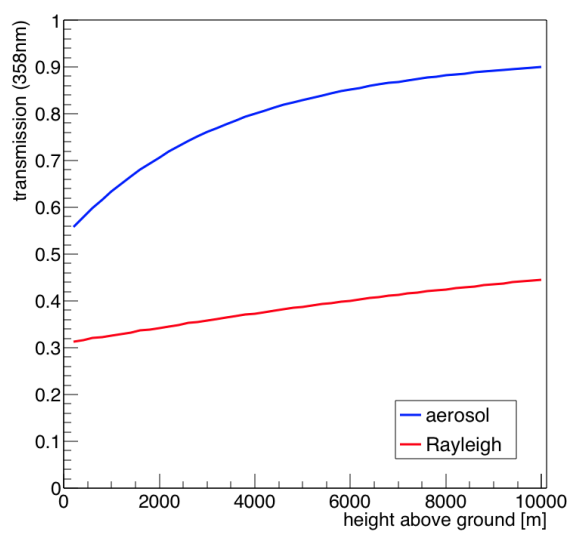

Figure 1. For a vertical shower at $20 \mathrm{~km}$ distance, we show the Rayleigh and aerosol transmission factors from a point on the shower axis (specified by the height above ground, here at 1400 m.a.s.l.) to the FD. For a wavelength of $358 \mathrm{~nm}$ (a particular fluorescence band), and $\operatorname{VAOD}(3 \mathrm{~km})=0.04$, a typical value. 
aerosol concentrations can change rapidly with time. The vertical distribution of aerosols determines the shape of the blue line in Figure 1; if this is measured incorrectly, it can not only affect energy measurements, but also the shape of the shower's longitudinal profile, and hence the measurement of the depth of maximum $X_{\max }$. A program of aerosol monitoring is therefore of key importance.

\section{Measurements of aerosols}

Auger's primary tools for measuring the effect of aerosols are the central laser facilities (the CLF and XLF) [4]. These facilities fire $6.5 \mathrm{~mJ}$ pulses of $355 \mathrm{~nm}$ laser light vertically into the atmosphere, and the scattered light is detected at the four FD sites $26 \mathrm{~km}$ to $30 \mathrm{~km}$ away. This coupling of vertical lasers and FD telescopes makes a series of bi-static lidar systems capable of determining the vertical distribution of aerosol attenuation. Two different analysis methods are used to extract the vertical aerosol optical depth as a function of height above the ground, $\operatorname{VAOD}(h)$ the so-called Data Normalised (DN) and Laser Simulation (LS) methods $[5,6]$.

The starting point for both methods is the measured light signal as a function of elevation in the FD telescope, and with a similar profile on a "reference Rayleigh night", a night with an atmosphere assumed to be free of aerosols. The DN method uses the ratio of the two light profiles to derive $\operatorname{VAOD}(h)$ and the derivative of the optical depth known as $\alpha(h)$, the aerosol scattering coefficient (units $\mathrm{m}^{-1}$ ). A recent improvement to the algorithm corrects an earlier simplification that assumed that light was scattered out of the laser beam by the Rayleigh process only, and another improvement takes account of a contribution of multiple-scattered light to the measured signal $[7,8]$.

The LS method finds the best match between the measured light profile and a library of simulated profiles, generated with a variety of aerosol density profiles. In the LS method the reference night light profile is used to normalise the simulations.

A series of 50 vertical laser shots is fired every 15 minutes during FD operation from both the CLF and the XLF, and analysis is performed to produce hourlyaveraged measurements of $\operatorname{VAOD}(h)$ and $\alpha(h)$ for each FD station. Roughly $90 \%$ of hours use the DN approach, with the remainder coming from the LS analysis.

\subsection{Characteristics of aerosols measured at Auger}

The distribution of VAOD at $3.5 \mathrm{~km}$ above ground level is shown in Fig. 2 for a period of seven years from three FD sites. The average value of this quantity is approximately 0.04 , and several physics analyses (e.g. energy, $X_{\max }$ ) apply a cut requiring this quantity to be below 0.1 .

The wavelength dependence of the aerosol attenuation has been measured at the Auger site along a $45 \mathrm{~km}$ path using a broad spectrum light source and a CCD camera (the Horizontal Attenuation Monitor, HAM [9]). The results are consistent with a weak wavelength dependence of $\lambda^{-\gamma}$ with an Angstrom coefficient $\gamma=0.7 \pm 0.5$. A fixed value

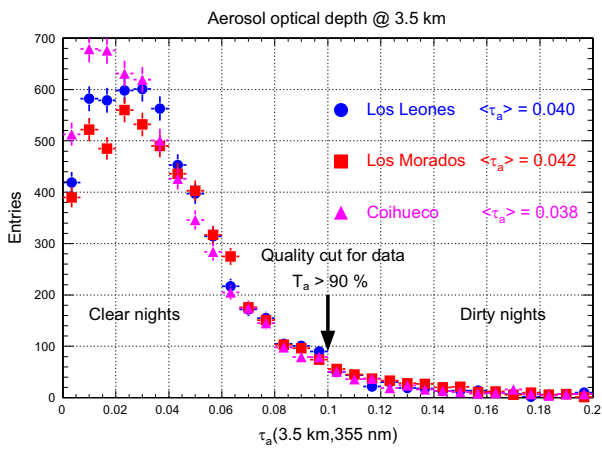

Figure 2. Distribution of VAOD $3.5 \mathrm{~km}$ above ground at $355 \mathrm{~nm}$ from three Auger sites over a period of 7 years [1]. Most physics analyses require this quantity to be $<0.1$.

of $\gamma=0.7$ is used in analysis (see below for the resulting systematic uncertainty in shower energy).

As explained below, another important parameter of aerosol scattering is its angular dependence, described by the normalised differential scattering cross-section (or "phase function") $P(\theta)=\sigma^{-1} d \sigma / d \Omega$, the probability per unit solid angle of a scattering angle of $\theta$. In analysis, we assume a form of the phase function known as a modified Henyey-Greenstein function (see [9]) with two parameters determining the strength of forward and back-scattering. Experimentally, we have observed the phase function using two aerosol phase-function monitors (APFs) near the Coihueco and Los Morados FD sites. An APF is a collimated xenon flash lamp which fires a light pulse horizontally across the field of view of the FD telescopes. The telescopes then measure the scattered light at scattering angles ranging from $30^{\circ}$ to $150^{\circ}$. The measured intensity as a function of angle can be fitted with the sum of Rayleigh and aerosol phase functions, see Figure 3. The implied aerosol phase function is well fitted by the modified Henyey-Greenstein function, and in analysis we use fixed values of its parameters, $f=0.4$ and $g=0.6$, derived from the means of the measured distributions [10].

\section{Reconstruction of shower energy, and depth of shower maximum}

The various steps for the reconstruction of a hybrid air shower are described in [1]. After the geometry of the shower axis is determined with the help of timing information from the SD station with the largest signal, the longitudinal development profile is constructed using the light collected at the FD as a function of time. That light at the detector consists of four main components: fluorescence light, direct Cherenkov light, and Cherenkov light scattered by the Rayleigh process and by aerosols. The production of fluorescence and Cherenkov light is fundamentally connected, and an analytical approach has been developed to use all the measured light to derive the longitudinal energy deposit profile $d E / d X(X)$ [11].

Fluorescence light production per unit depth of atmosphere is directly proportional to the energy deposited by the air shower per unit depth, $w_{i}$ (using the same notation as [11]). The Cherenkov light production in a given 

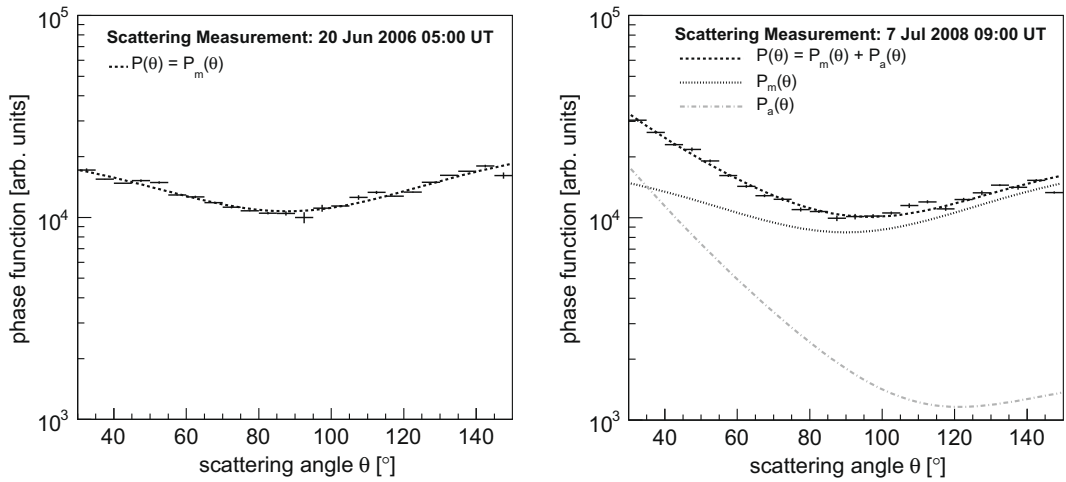

Figure 3. APF light intensity as a function of scattering angle for two nights, one essentially aerosol-free (left), and one more typical (right) [9]. Data are fitted with a sum of phase functions: molecular (Rayleigh) $P_{m}(\theta)$ and aerosol $P_{a}(\theta)$. Note the predominant forward-scattering peak in $P_{a}(\theta)$ in the right-hand plot.

depth interval is proportional to the number of electrons and positrons with energies above the Cherenkov threshold energy, $N_{i}^{c}$. Importantly, $w_{i}$ and $N_{i}^{c}$ are related by a function describing the energy loss of an electron of energy $E$, and the energy distribution of electrons as a function of shower age, both straightforward to evaluate with very little model dependence (see [11] for references).

Each of the four light components also have an angular dependence for emission (including the isotropic emission of the fluorescence). The angular distributions of scattered Cherenkov light are governed by the differential scattering cross-sections (phase functions) related to Rayleigh and aerosol scattering. We use the Rayleigh phase function from Bucholtz $[9,12]$ and the modified Henyey-Greenstein function for aerosols (section 2.1).

The goal of the profile reconstruction is to explain the received light at the telescope as the sum of these four components, thus yielding a solution for the energy deposit profile along the shower axis, $d E / d X(X)$. The aerosol information is used in two ways. It is used to calculate the aerosol attenuation of the light along various paths (e.g. from the shower axis to the telescope, or the attenuation of the growing Cherenkov beam along the shower axis). The aerosol scattering coefficient $\alpha$ and the phase function are also used to calculate the amount of aerosol-scattered Cherenkov light expected at the telescope from a particular point. An example (rather nice) shower profile is shown in Figure 4. The reconstructed $d E / d X(X)$ points are fitted with a Gaisser-Hillas (G-H) profile to take account of energy deposit outside the telescope field of view. The G-H function is then integrated over depth to get the "calorimetric" energy of the cascade, and the peak of the profile is $X_{\max }$ used in masscomposition studies. The primary cosmic ray energy is determined by adding a small $(\sim 15 \%)$ "invisible" energy correction to the calorimetric energy [13].

\section{Statistical and systematic uncertainties}

\subsection{Errors relating to VAOD}

The measured laser light profiles are accompanied with "correlated" and "uncorrelated" errors (listed in Table 1),

\begin{tabular}{|l|c|c|}
\hline & Correlated & Uncorrelated \\
\hline Relative FD calibration & $2 \%$ & $4 \%$ \\
Relative laser energy (CLF) & $1-2.5 \%$ & $2 \%$ \\
Relative laser energy (XLF) & $1 \%$ & $2 \%$ \\
Reference clean night & $3 \%$ & - \\
Atmospheric variations & - & $\sim 3 \%$ \\
\hline
\end{tabular}

Table 1. Errors relevant to the calculation of VAOD from CLF/XLF laser profiles at the FD. See text and [14] for details.

which propagate through to systematic and statistical errors in the VAOD, and to quantities such as shower energy and $X_{\max }$. Correlated errors are correlated across a sample of EAS, while uncorrelated errors could vary in magnitude and sign from one EAS to the next.

Since both methods for measuring the VAOD (DN and LS) use a reference night, we are not sensitive to systematic errors in the absolute laser or FD calibration, but we must take care of possible drifts in the relative calibrations between the reference night and the night in question. This is the origin of the "correlated" errors in the first three rows of Table 1. The "uncorrelated" errors for these rows relate to statistical uncertainties in the FD calibration during the night, and of the laser pulse energy. A 3\% correlated error relates to the statistical uncertainty of the light profile on the reference night; and an additional uncorrelated error relates to the statistical variation of the four quarter-hour average light profiles taken over a given hour. This is measured and is typically $\sim 3 \%$. See [14] for a plot of a typical VAOD profile including correlated and uncorrelated errors.

The correlated VAOD errors lead to a systematic error in shower energy which is energy dependent, ranging from $3-6 \%$ (from $10^{18} \mathrm{eV}$ to the highest energies). The uncorrelated VAOD errors also lead to a $3-6 \%$ contribution to the resolution of energy measurements [15].

\subsection{Other aerosol uncertainties}

Other aerosol-related uncertainties have also been propagated through to systematic uncertainties in shower energy. Uncertainties in the shape of the aerosol phase func- 

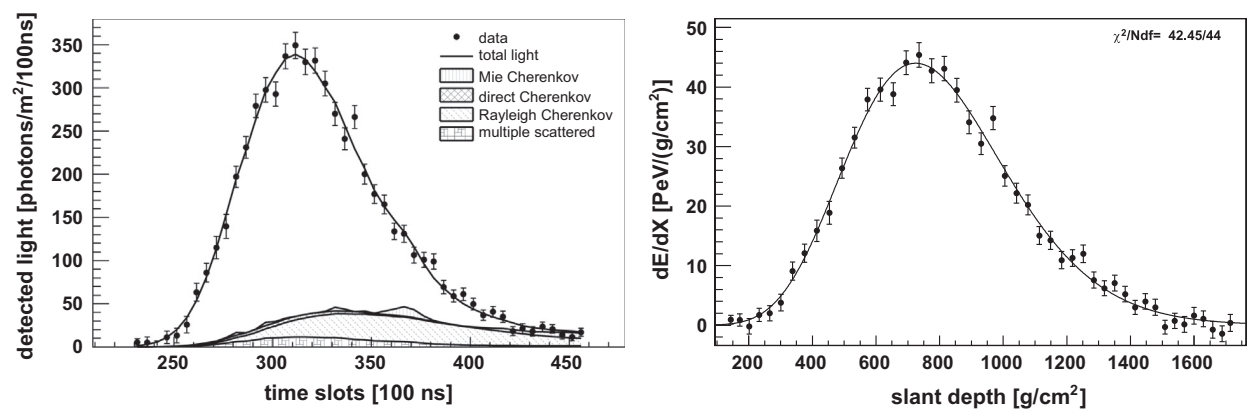

Figure 4. An example of shower profile reconstruction. The left plot shows the light received at the telescope aperture as a function of time, with the reconstructed direct and scattered Cherenkov contributions indicated, as well as a small contribution of multiple-scattered light. The difference between the "total" and the named components is the reconstructed fluorescence light at the aperture. On the right is the reconstructed energy deposit profile, together with a fit of the Gaisser-Hillas function. From [1].

tion lead to a systematic of $1 \%$, and uncertainties in the wavelength dependence of aerosol scattering contribute a systematic of only $0.5 \%$. All of the aerosol uncertainties mentioned here contribute to the overall systematic uncertainty in energy (along with calibration, fluorescence yield, reconstruction etc.) of $14 \%$, and a typical statistical uncertainty of $17-12 \%$ (energy dependent) [15].

For $X_{\max }$, the systematic effects of all atmospheric parameters have been combined, and are shown as a function of energy in Figure 5. The atmosphere is the dominating source of the systematic at the highest energies, contributing between approximately -4 and $+8 \mathrm{~g} / \mathrm{cm}^{2}$. The aerosol contribution to the $X_{\max }$ resolution is up to $10 \mathrm{~g} / \mathrm{cm}^{2}$ at the highest energies, a significant contributor to the total resolution of $15 \mathrm{~g} / \mathrm{cm}^{2}$ at those energies [16].

\section{Conclusions}

We have discussed the primary techniques used to characterise the distribution of light-attenuating aerosol particles above the Pierre Auger Observatory, and how that information is used to correct fluorescence detector observations of EAS energy and $X_{\max }$. Unfortunately, it has not been possible to describe other techniques used at the Observatory for aerosol characterisation. Luckily, two of those methods, the Raman Lidar and FRAM, are being presented separately at this conference $[17,18]$.

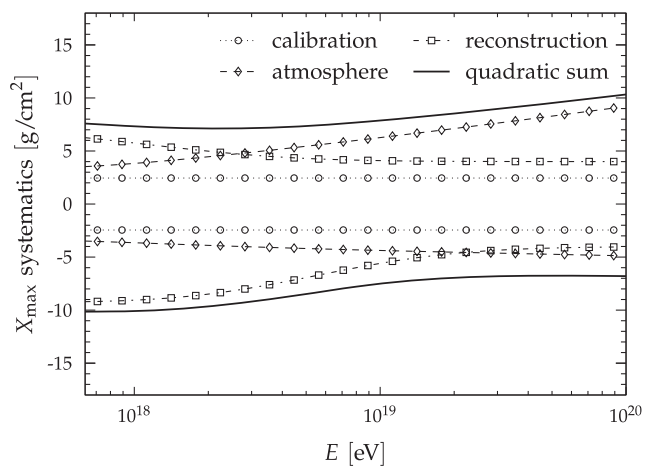

Figure 5. Systematic uncertainty in $X_{\max }$ as a function of energy. The aerosol uncertainties are the major part of the curves labelled "atmosphere" [16].

\section{References}

[1] A. Aab et al. [The Pierre Auger Collaboration], Nuclear Instruments and Methods A 798, 172 (2015)

[2] M. Ave, M. Bohacova, E. Curry et al., Astroparticle Physics 42, 90 (2013)

[3] P. Abreu et al. [The Pierre Auger Collaboration], Astroparticle Physics 35, 591 (2012)

[4] B. Fick, M. Malek, J.A.J. Matthews et al., Journal of Instrumentation 1, P11003 (2006)

[5] P. Abreu et al. [The Pierre Auger Collaboration], Journal of Instrumentation 8, P04009 (2013)

[6] L. Valore, this conference (2018)

[7] M. Malacari, for the Pierre Auger Collaboration, PoS ICRC2017, 398 (2018)

[8] M. Malacari, B.R. Dawson, Astroparticle Physics 93, 38 (2017)

[9] J. Abraham et al. [The Pierre Auger Collaboration], Astroparticle Physics 33, 108 (2010)

[10] S. BenZvi, B. Connolly, J. Matthews et al., Astroparticle Physics 28, 312 (2007)

[11] M. Unger, B. Dawson, R. Engel et al., Nuclear Instruments and Methods A 588, 433 (2008)

[12] A. Bucholtz, Appl. Opt. 34, 2765 (1995)

[13] A. Mariazzi, M. Tueros, for the Pierre Auger Collaboration, JSPS Conf. Proc. 19, 011006 (2016)

[14] L. Valore, for Pierre Auger Collaboration, in Proceedings, 33rd International Cosmic Ray Conference (2013), p. 0920, arXiv: 1307.5059

[15] V. Verzi, for Pierre Auger Collaboration, in Proceedings, 33rd International Cosmic Ray Conference (2013), p. 0928, arXiv: 1307.5059

[16] A. Aab et al. [The Pierre Auger Collaboration], Phys. Rev. D 90, 122005 (2014)

[17] V. Rizi, this conference (2018)

[18] J. Ebr, this conference (2018) 